\title{
Electrochemical Behavior of a Pd Thin Film Electrode in Concentrated Alkaline Media
}

\author{
K. Hubkowska ${ }^{1}$ (D) M. Soszko ${ }^{2}$ - M. Symonowicz ${ }^{1}$ M. Lukaszewski ${ }^{1}$ \\ A. Czerwiński ${ }^{1,2,3}$
}

Published online: 22 April 2017

(C) The Author(s) 2017. This article is an open access publication

\begin{abstract}
Pd limited volume electrodes (LVE) were obtained electrochemically from the $\mathrm{PdCl}_{2}$ aqueous solution. Hydrogen absorption was performed using cyclic voltammetry and chronoamperometry in concentrated $(6 \mathrm{M})$ alkaline solutions $(\mathrm{KOH}, \mathrm{NaOH}$, the mixture of $2 \mathrm{M} \mathrm{LiOH}+4 \mathrm{M} \mathrm{KOH})$ and results were compared with $0.5 \mathrm{M} \mathrm{H}_{2} \mathrm{SO}_{4}$. It was found that hydrogen pretreatment procedure is crucial for further examination of hydrogen absorption in Pd electrodes. After many cycles of hydrogen absorption/desorption in Pd, these processes become electrochemically more reversible - faster than in a freshly prepared electrode. Results obtained for Pd electrodes in different concentrated alkaline media subjected to hydrogen pretreatment procedure are comparable with those for an acidic solution (e.g., $\alpha \rightarrow \beta$ phase transition potential equals to ca. $0.050 \mathrm{~V}$ vs. RHE). The continuous Pd electrode cycling does not affect significantly the amount of absorbed hydrogen (ca. 0.72-0.74). The surface roughness factor in basic solutions decreases after multiple hydrogen sorption as in case of an acidic solution.
\end{abstract}

Keywords Pd limited volume electrode $\cdot$ Hydrogen sorption · $\alpha \leftrightarrow \beta$ phase transition potential $\cdot$ Hydrogen pretreatment procedure $\cdot$ Alkaline solutions

K. Hubkowska

khubkowska@chem.uw.edu.pl

1 Faculty of Chemistry, University of Warsaw, Pasteura 1, 02-093 Warsaw, Poland

2 Industrial Chemistry Research Institute, Rydygiera 8, 01-793 Warsaw, Poland

3 Faculty of Chemistry, Biological and Chemical Research Centre, University of Warsaw, Żwirki i Wigury 101, 02-089 Warsaw, Poland

\section{Introduction}

Since Graham published his findings in 1866 [1], palladium properties have been thoroughly examined mainly due to its ability to hydrogen absorption. Nevertheless, Pd behavior in many aspects requires further research and explanation. In literature, there are many reports concerning Pd properties in acidic solutions [2-9] but there are relatively very few articles referring to Pd properties in basic solutions, especially in concentrated media.

Martin and Lasia studied hydrogen absorption and adsorption in/on Pd monolayers in $0.1 \mathrm{M} \mathrm{NaOH}$ solutions. They pointed that the addition of benzotriazole (BTA) promotes kinetically absorption and inhibits adsorption of hydrogen [10]. The same research group stated that hydrogen sorption kinetics in Pd (several dozen nm thick) is inhibited in basic solutions in Pd as compared with acidic media and therefore hydrogen sorption isotherms are shifted into lower potential values [11]. Palladium $30 \mathrm{~nm}$ thick films obtained by pulsed layer deposition (PLD) were examined in the aspect of hydrogen sorption from $1 \mathrm{M} \mathrm{KOH}$ solution by Paillier and Roué [12]. The authors indicate that the kinetics of hydrogen sorption was facilitated with the decrease in Pd thickness; however, the amount of absorbed hydrogen does not exceed the values reported for coarse-grained films. In case of films obtained by PLD, there was no flat plateau of the $\alpha \leftrightarrow \beta$ phase transition, which is ascribed by Pallier and Roué to the wide distribution of energy levels in Pd thin films, being the main reason of good cyclic stability. Impedance analysis of hydrogen absorption in a Pd foil in $0.1 \mathrm{M} \mathrm{NaOH}$ was presented by Yang and Pyun [13]. The authors suggest that the phase boundary between the $\alpha$ - and $\beta$-phase hinders the hydrogen diffusion at palladium during charging with hydrogen. It was also stated that the $\beta$-phase of hydrogen formed near the electrode surface behaves as a barrier for hydrogen diffusion in the 
potentials below $0.08 \mathrm{~V}$ (vs RHE), where direct hydrogen absorption reaction (har) is postulated. Rubes and Bilyková [14] showed the connection between catalytic activity of Pd and the amount of absorbed hydrogen in $6 \mathrm{M} \mathrm{KOH} .0 .3 \%$ at. hydrogen in Pd appeared to be optimal in case of $\mathrm{HCOO}^{-}$ oxidation, while increasing hydrogen content in $\mathrm{Pd}$ resulted in a drop in its catalytic activity. $\mathrm{Hu}$ and Wen [15] from the experiment of hydrogen absorption in $1 \mathrm{M} \mathrm{NaOH}$ in oxidederived Pd in the presence of p-nitroaniline noticed that this organic compound reacts only with adsorbed hydrogen, which indicates that the $\beta$-phase of hydrogen is not formed from the $\alpha$-phase. Thorough examination of oxide-derived Pd electrodes in acidic and basic media in comparison with Pd-bulk electrodes gives the ability to ascertain that the surface roughness of electrodes polarized in basic media is higher than in an acid [16]. The authors also postulate that the utilization of a Pd-oxide derived electrode enables to analyze separately signals originating from hydrogen adsorption and absorption, in contrast to a Pd bulk electrode. The electrochemistry of a palladized Pd electrode was examined by Burke and Casey mainly with the emphasis on the premonolayer oxidation [17]. It was found that on disordered surfaces there are a lot of sites where hydrous oxide mediators can be generated. The same group also stated that hydrogen absorption in activated $\mathrm{Pd}$ causes the activation of surface and results in the formation of recalcitrant hydrous oxides [18]. Czerwiński's group studied the problem of alkaline metal insertion into Pd from $0.1 \mathrm{M}$ basic solutions [19-21]. They noticed that alkaline metals influence the $\alpha \rightarrow \beta$ phase transition potential, which is connected with alkaline metals incorporation into the bulk of Pd at lower potentials during hydrogen electrolysis. This effect was not observed in neutral and acid solutions with alkali cations [21].

Studying Pd properties in concentrated alkaline solutions is important not only in the aspect of basic research, but also in the application aspect, since Pd is still considered as a small addition [22] to commercially used alloys in hydride batteries [23-26]. Pd addition results in lower charge/discharge overpotentials, lower charge transfer resistance and improved cycle life [25]. Modification of the alloys with the palladium also accelerates the activation process and enhances the ratecapability $[24,26]$.

In this short paper, Pd behavior in concentrated $(6 \mathrm{M})$ basic solutions of $\mathrm{KOH}, \mathrm{NaOH}$ and the mixture of $\mathrm{LiOH}$ and $\mathrm{KOH}$ is presented (in comparison with $0.5 \mathrm{M} \mathrm{H}_{2} \mathrm{SO}_{4}$ solution). We describe the influence of the process of hydrogen pretreatment procedure on the hydrogen absorption properties of Pd thin films, namely the value of potential of hydrogen oxidation, the amount of absorbed hydrogen and the $\alpha \rightarrow \beta$ phase transition potentials with respect to our previous works focused on electrochemical behavior of Pd thin film electrodes in acidic electrolytes.

\section{Experimental}

Pd limited volume electrodes $(0.5 \mu \mathrm{m}$ thick) were deposited potentiostatically (deposition potential $0.36 \mathrm{~V}$ vs SHE) on a gold wire $(0.5 \mathrm{~mm}$ diameter) from the solution of $0.11 \mathrm{M}$ $\mathrm{PdCl}_{2}$ in $1 \mathrm{M} \mathrm{HCl}$. After deposition, the electrodes were subjected to hydrogen pretreatment procedure consisting of the following steps: (1) one $\mathrm{CV}$ cycle in the potential range $0.63 \mathrm{~V} \div-0.37 \mathrm{~V}$ vs. RHE, scan rate: $0.01 \mathrm{Vs}^{-1}$; (2) $50 \mathrm{CA}$ steps (hydrogen absorption/desorption potentials: $-0.37 \mathrm{~V} /$ $0.63 \mathrm{~V}$ ) with $200 \mathrm{~s}$ pulse width; (3) $50 \mathrm{CV}$ cycles in potential range $0.63 \mathrm{~V} \div-0.37 \mathrm{~V}$; (4) alternately second and third procedure to obtain $14 \mathrm{CA}$ and $14 \mathrm{CV}$ procedures. During the pretreatment procedure, the solution was purged with argon (Air Products, 99.999\% purity), removing gaseous oxygen and hydrogen, and simultaneously mixing the solution near the working electrode surface (without the impact on the registered curves). Before other electrochemical measurements were performed, the solution was purged with Ar for $20 \mathrm{~min}$, while during the measurements Ar stream floated above the solution. The measurements were carried out in a three-electrode system with a Pd-LVE working electrode, an $\mathrm{Hg} / \mathrm{HgO} / 6 \mathrm{M} \mathrm{KOH}$ (or in case of the acidic electrolyte: $\mathrm{Hg}$ / $\mathrm{Hg}_{2} \mathrm{SO}_{4} / 0.5 \mathrm{M} \mathrm{H}_{2} \mathrm{SO}_{4}$ ) reference electrode and a $\mathrm{Pt}$ gauze auxiliary electrode. $\mathrm{CH}$ Instruments model 760D was utilized in electrochemical measurements. Analytical grade reagents (Avantor, Sigma-Aldrich, Alfa-Aesar) and water purified in Hydrolab and Millipore systems were used to prepare all the solutions. The purity of the reagents used to prepare electrolytes: KOH: PA grade, assay $\geq 85$ (Avantor), $\mathrm{NaOH}$ : PA grade, assay $\geq 98.8 \%$ (Avantor), LiOH: ACS reagent grade, assay $\geq 98 \%$ (Sigma-Aldrich).

The potentials were recalculated with respect to the reversible hydrogen electrode (RHE) in the working solution with the use of Hydroflex Hydrogen Reference Electrode purchased from Gaskatel GmbH, Germany. All measurements were done in the temperature of $298 \mathrm{~K}$ controlled by a thermostat Lauda Eco Re 630. The parameter of $H / P d$ was calculated on the basis of the charge obtained from the integration of the hydrogen oxidation cyclic voltammetry signals and the deposition charge of Pd.

\section{Results and Discussion}

Figure 1 shows a comparison of $\mathrm{CV}$ curves recorded in a wide potential range for a Pd-LVE in $6 \mathrm{M} \mathrm{KOH}$ solution at two stages of the electrochemical treatment: (1) for a freshly deposited Pd, i.e., not subjected earlier to any kind of hydrogen sorption (blue line), (2) for the same electrode after prolonged hydrogen absorption/desorption procedure (black line). The insert shows the potential region of surface oxides formation/reduction. 


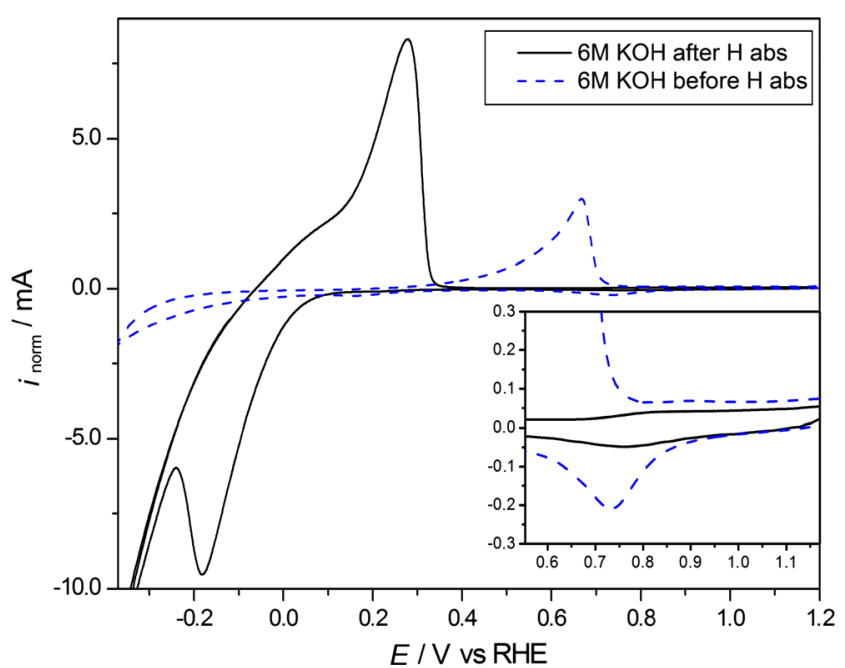

Fig. 1 Cyclic voltammetry of a Pd-LVE electrode (scan rate $0.1 \mathrm{Vs}^{-1}$ ) in the potential range $-0.4 \mathrm{~V} \div 1.25 \mathrm{~V}$ in $6 \mathrm{M} \mathrm{KOH}$ before and after multiple hydrogen electrochemical sorption

The course of the CV curve for the electrode after the electrochemical treatment differs markedly from that for the fresh Pd deposit. The following facts should be noted: (1) hydrogen absorption/desorption signals (i.e., peaks placed below $0.4 \mathrm{~V}$ ) are higher, sharper and both cathodic and anodic peaks are well developed in contrast to a poorly defined cathodic signal before the pretreatment, (2) the potential difference in the position of hydrogen absorption and desorption signals in much smaller, (3) currents in the oxide region become lower.

The changes in the $\mathrm{CV}$ response after prolonged hydrogen absorption/desorption in $6 \mathrm{M} \mathrm{KOH}$ electrolyte are qualitatively identical to those reported earlier for a Pd electrode pretreatment in acidic solutions [27]. This behavior indicates that after many cycles of hydrogen absorption/desorption in Pd electrodeposits these processes become electrochemically more reversible, i.e. faster than in a freshly prepared electrode. In the literature, the improved kinetics of hydrogen electrosorption after hydrogen treatment was explained by an increase in the number of defects in Pd crystal lattice (caused by hydrogen penetration), the presence of which facilitates further hydrogen transport within the Pd bulk [28]. Successive hydrogen absorption/desorption cycles may also release the stresses in the metal and facilitate the processes of the $\alpha \rightarrow \beta$ and $\beta \rightarrow \alpha$ phase transitions, and their rate influences the overall rate of the electrosorption process.

From Fig. 1, it can be noted that hydrogen oxidation charges differ in case of $\mathrm{CV}$ curves before and after hydrogen pretreatment procedure. In case of the Pd electrode before hydrogen absorption, the scan rate of $0.1 \mathrm{Vs}^{-1}$ is too high to obtain full saturation of hydrogen in measured potential range. In case of Pd electrode after multiple hydrogen absorption, the process of hydrogen sorption becomes faster and full saturation of hydrogen is completed at higher potential values, therefore absorption with the scan rate of $0.1 \mathrm{Vs}^{-1}$ gives the ability to absorb higher amount of hydrogen than in case of the Pd fresh electrode.

The lowering currents in the surface oxide formation/ reduction potential region suggest the decrease in the electrode real surface area. Indeed, the values of the roughness factor (defined as the ratio of the real surface area to the geometric area of the electrode), as calculated from the charges of surface oxide reduction [29], markedly decreased after the sorption procedure, i.e., from 29.5 to 6.6 . The same trend was observed for other electrolytes, namely $6 \mathrm{M} \mathrm{NaOH}$ (a decrease from 42.1 to 11.5 ) and $6 \mathrm{M} \mathrm{LiOH}$ (from 34 to 3.5). Again, this tendency in the surface roughness changes is in line with the observations made earlier for acidic solutions. The analysis of this behavior has been discussed in our previous papers [30].

Figure 2 shows the evolution of CVs during the aforementioned procedure of hydrogen treatment of a Pd-LVE in $6 \mathrm{M}$ $\mathrm{KOH}$. It clearly demonstrates the development and shift of the hydrogen absorption peak to higher potentials (from ca. -0.15 to $0.0 \mathrm{~V}$ ), together with the shift of the hydrogen desorption signal to lower potentials (from ca. 0.30 to $0.1 \mathrm{~V}$ ), resulting in a significant decrease in the potential difference between the position of the cathodic and anodic hydrogen peaks. Such situation has already been reported for Pd-LVEs in acidic solutions [27].

Figure 3 shows a superposition of CV curves $(0.01 \mathrm{~V} / \mathrm{s})$ recorded for Pd-LVEs after the hydrogen treatment in acidic and basic electrolytes, i.e., $0.5 \mathrm{M} \mathrm{H}_{2} \mathrm{SO}_{4}$ (red line) and $6 \mathrm{M}$ $\mathrm{KOH}$ (blue line). It should be noted that the general course and position of these curves on the potential axis in the RHE scale are very similar. The most important difference concerns the potentials of the anodic peaks due to hydrogen oxidation,

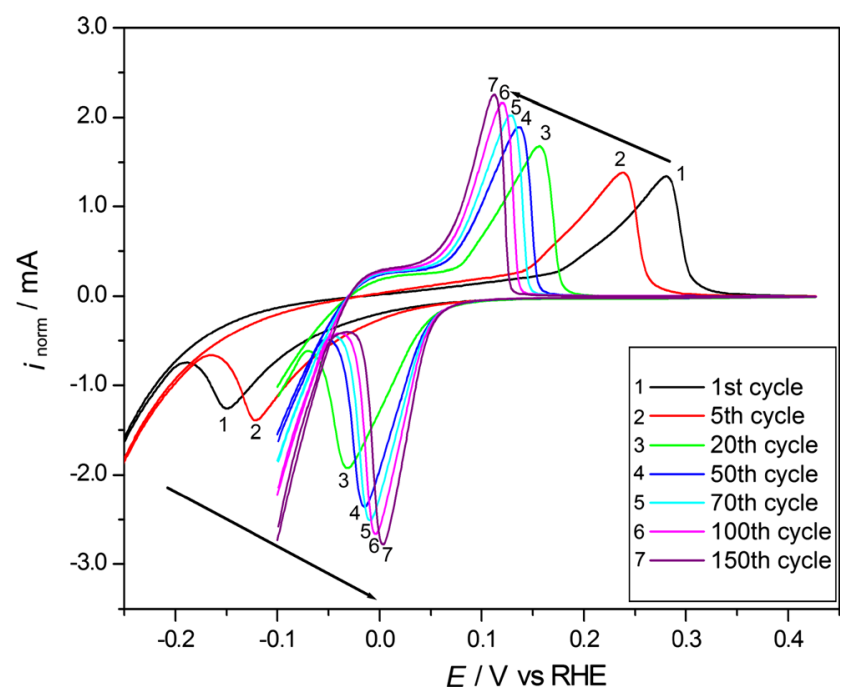

Fig. 2 Selected cyclic voltammetry curves of a Pd-LVE electrode (scan rate $0.01 \mathrm{Vs}^{-1}$ ) in the potential range $-0.3 \mathrm{~V} \div 0.45 \mathrm{~V}$ in $6 \mathrm{M} \mathrm{KOH}$ registered during the hydrogen pretreatment procedure 


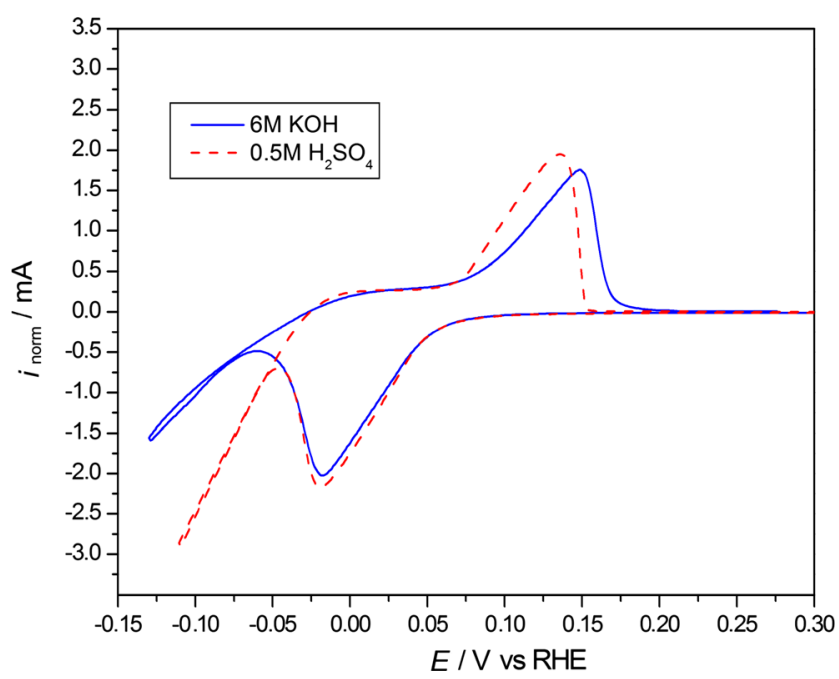

Fig. 3 Comparison of cyclic voltammetry curves of a Pd-LVE electrode (scan rate $0.01 \mathrm{Vs}^{-1}$ ) in the potential range $-0.13 \mathrm{~V} \div 0.4 \mathrm{~V}$ in $0.5 \mathrm{M}$ $\mathrm{H}_{2} \mathrm{SO}_{4}$ and $6 \mathrm{M} \mathrm{KOH}$ after the hydrogen pretreatment procedure

which in the acidic solution is placed at a potential lower than the same signal in the basic solution (i.e. at ca. 0.13 vs. $0.15 \mathrm{~V}$ ), although no such difference is observed in the case of the cathodic peaks due to hydrogen absorption (both placed at ca. $-0.02 \mathrm{~V}$ ). The greater potential difference between cathodic and anodic hydrogen peaks in bases mirrors the already known fact of a higher electrochemical irreversibility of the hydrogen absorption/desorption processes in basic solutions as compared to the behavior in acidic electrolytes. The literature data confirm the slower rate of hydrogen electrosorption in Pd from various basic solutions [11].

Figure 4 indicates the fact that the procedure of the hydrogen treatment requires more hydrogen absorption/desorption cycles to be performed in basic electrolytes than in an acid.

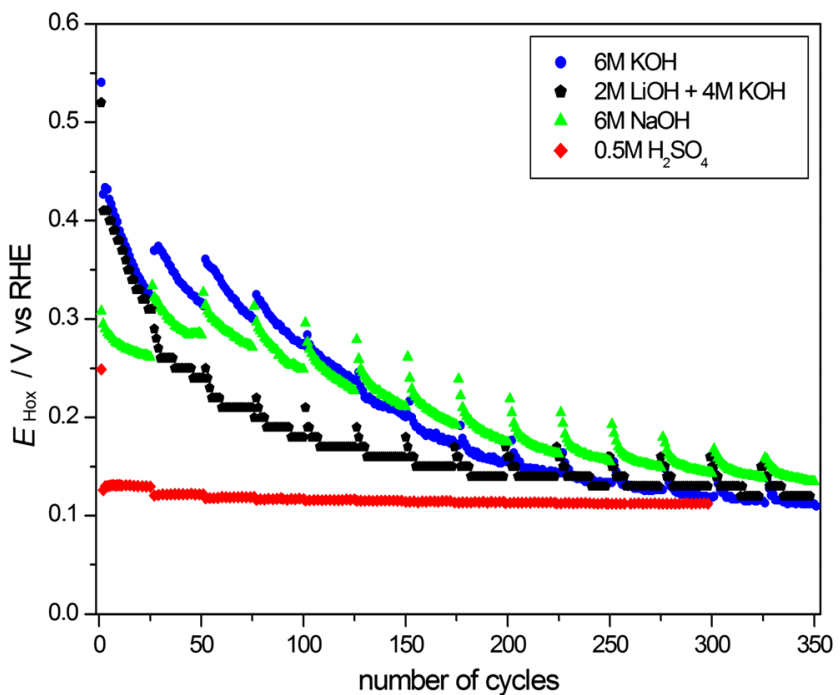

Fig. 4 Dependence of the hydrogen oxidation peak potential on the number of cycles calculated from the $\mathrm{CV}$ curves (scan rate $0.01 \mathrm{Vs}^{-1}$ ) registered during the hydrogen pretreatment procedure for different electrolyte composition
Typically, a steady-state CV (mirrored in a constant position of hydrogen peaks) in an acid $\left(0.5 \mathrm{M} \mathrm{H}_{2} \mathrm{SO}_{4}\right)$ can be obtained already after several tenths of cycles, while in all basic solutions studied here $(6 \mathrm{M} \mathrm{KOH}, 6 \mathrm{M} \mathrm{NaOH}$ and a mixture of $2 \mathrm{M} \mathrm{LiOH}+4 \mathrm{M} \mathrm{KOH}$ ) no less than ca. 300 cycles were required for the $\mathrm{CV}$ stabilization.

In order to study whether the procedure of hydrogen treatment influences only the kinetics of the electrosorption process and/or also affects the total amount of absorbed hydrogen, the values of the hydrogen-to-palladium atomic ratio $(H / P d)$ were determined at each stage of the pretreatment procedure. Figure 5 shows that there is a rather little effect of the number of hydrogen absorption/desorption cycles on the absorption capacity of Pd-LVEs in both basic and acidic electrolytes. Therefore, it can be suggested that the hydrogen treatment procedure affects mainly the kinetics but it has a much smaller influence on the maximum amount of hydrogen electrosorbed in Pd-LVEs. At this stage of studies, it is difficult to state the reason for the decrease in $H / P d$ ratios after longer cycling in some of the electrolytes examined and for the absence of this effect in others.

In Fig. 6, the values of the $H / P d$ ratio vs. electrode potential are plotted for four different electrolytes. These data concern the Pd-LVEs after the completion of the pretreatment procedure and all the values of $H / P d$ represent stationary values corresponding to the electrode full saturation with hydrogen at each potential. One can see that all the curves almost fully coincide within the entire potential range in the RHE scale. In particular, the alpha-beta phase transition is placed at ca. $0.050 \mathrm{~V}$ vs. RHE in all cases, with only small deviations (within ca. $0.005 \mathrm{~V}$ ) for a given electrolyte. The value of $\mathrm{E}_{\alpha \rightarrow \beta}$ deviation was estimated on the basis of several $H / P d$ ratio vs $E$ profiles plotted after multiple repeatable measurements.

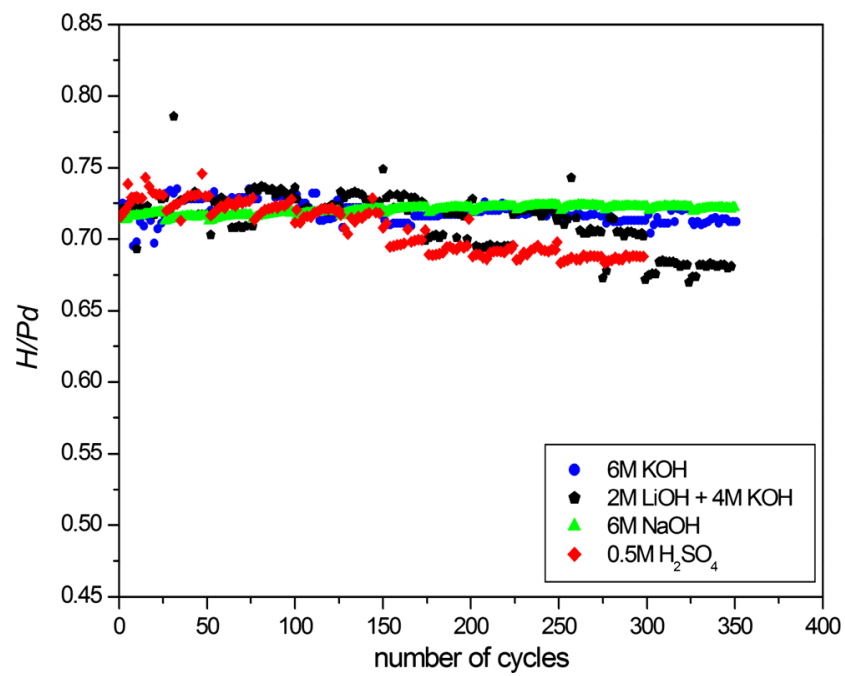

Fig. 5 Dependence of the amount of absorbed hydrogen (expressed as hydrogen-to-metal atomic ratio: $H / P d$ ) on the number of cycles calculated from the $\mathrm{CV}$ curves (scan rate $0.01 \mathrm{Vs}^{-1}$ ) registered during the hydrogen pretreatment procedure for different electrolyte composition 


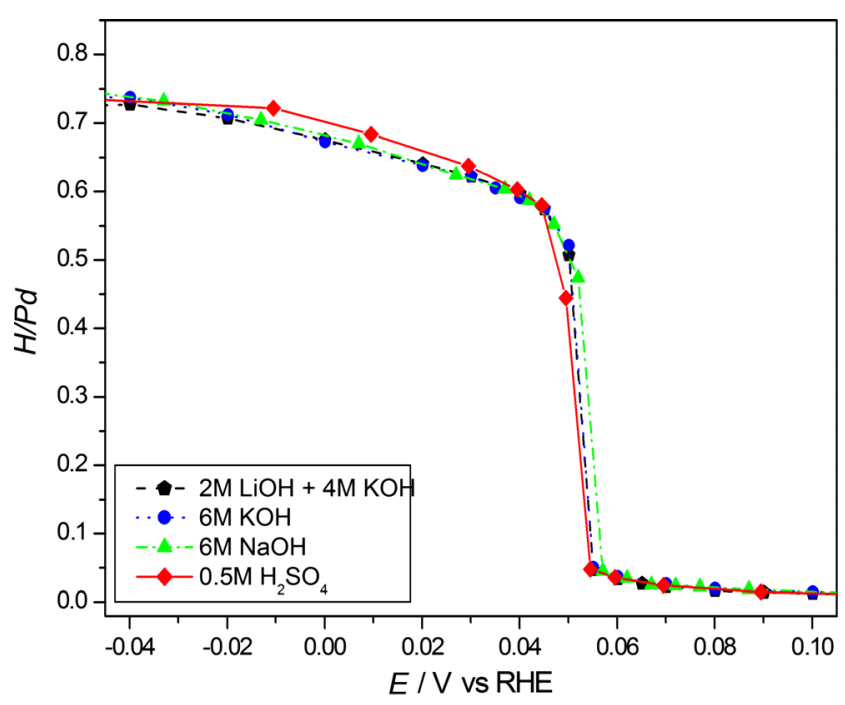

Fig. 6 Influence of the electrode potential and electrolyte composition on the amount of absorbed hydrogen $(H / P d)$ calculated from charges obtained from cyclic voltammetry curves (scan rate $0.01 \mathrm{Vs}^{-1}$ )

Also, the amounts of hydrogen electrosorbed in Pd-LVEs in the $\alpha$ and $\beta$ phases are practically the same for each solution studied. The maximum $H / P d$ values at the lowest edge of the potential range applied in these studies are ca. 0.73-0.74, regardless of the kind of the electrolyte, i.e., they are the same in both basic and acidic solutions.

Some earlier reports $[16,19]$ indicated that in basic solutions the maximum amount of hydrogen electrosorbed in Pd could be higher than that in acids, i.e. corresponding to $H / P d$ approaching or even exceeding 0.80 . However, in those cases, the following differences in experimental conditions might have been responsible for the different $H / P d$ values as compared to our results: (1) Pd electrodeposits had not been subjected to the hydrogen pretreatment, (2) hydrogen saturation was performed at potentials low enough for massive hydrogen gas evolution to occur, which could have contributed to the further measured amount of oxidized hydrogen, (3) the lack of electrolyte mixing during absorption/desorption of hydrogen.

A detailed analysis of the literature and our data for the behavior of Pd-LVEs prepared and pretreated in different ways and examined under various conditions of hydrogen electrosorption in basic solutions will be the subject of a separate paper.

\section{Conclusions}

The hydrogen pretreatment procedure plays a crucial role in the further examination of hydrogen sorption properties of Pd electrodes. The hydrogen pretreatment procedure is hindered in basic solutions in comparison with acid, therefore the electrode should be polarized long enough to obtain repeatable course of $\mathrm{CV}$ curves in subsequent $\mathrm{CV}$ cycles. In alkaline media, the surface roughness factor decreases after multiple hydrogen sorption as in case of acid solution. There is no difference between the course of CV curve (the shape and the position of hydrogen absorption and desorption signals) and $H / P d$ vs E curves after hydrogen pretreatment procedure in alkaline and acid media. The $\alpha \rightarrow \beta$ phase transition potential, regardless of the type of solution, equals to approx. $0.050 \mathrm{~V}$ vs RHE $( \pm 0.0025 \mathrm{~V})$. The continuous Pd electrode cycling does not affect the hydrogen capacity - there are no significant changes in the amount of absorbed hydrogen at the beginning and at the end of hydrogen pretreatment procedure.

Acknowledgements This research was funded from grant no. 2015/17/ B/ST8/03377 (ID 289956) of the Polish National Centre.

\section{Compliance with Ethical Standards}

Conflict of Interest The authors declare that they have no conflict of interest.

Open Access This article is distributed under the terms of the Creative Commons Attribution 4.0 International License (http:// creativecommons.org/licenses/by/4.0/), which permits unrestricted use, distribution, and reproduction in any medium, provided you give appropriate credit to the original author(s) and the source, provide a link to the Creative Commons license, and indicate if changes were made.

\section{References}

1. T. Graham, Philos. Trans. Roy. Soc. London 156, 399 (1866)

2. T.B. Flanagan, F.A. Lewis, Trans. Faraday Soc. 55, 1400 (1959)

3. H. Duncan, A. Lasia, Electrochim. Acta 52, 6195 (2007)

4. A. Czerwiński, J. Electroanal. Chem. Interfacial Electrochem. 316, $211(1991)$

5. M. Baldauf, D.M. Kolb, Electrochim. Acta 38, 2145 (1993)

6. L.D. Burke, J.K. Casey, J. Electrochem. Soc. 140, 1284 (1993)

7. G. Jerkiewicz, Prog. Surf. Sci. 57, 137 (1998)

8. A. Zalineeva, S. Baranton, C. Coutanceau, G. Jerkiewicz, Langmuir 31, 1605 (2015)

9. A. Zalineeva, S. Baranton, C. Coutanceau, G. Jerkiewicz, Sci. Adv. 3, e1600542 (2017)

10. M.H. Martin, A. Lasia, Electrochim. Acta 54, 5292 (2009)

11. M.H. Martin, A. Lasia, Electrochim. Acta 53, 6317 (2008)

12. J. Paillier, L. Roué, J. Electrochem. Soc. 152, E1 (2005)

13. T.H. Yang, S.I. Pyun, Electrochem. Acta 41, 843 (1996)

14. I. Rubeš, H. Bilyková, Collection. Czech. Chem. Commun. 37, 715 (1972)

15. C.C. Hu, T.C. Wen, J. Electrochem. Soc. 142, 1376 (1995)

16. C.C. Hu, T.C. Wen, J. Electrochem. Soc. 141, 2996 (1994)

17. L.D. Burke, J.K. Casey, J. Electrochem. Soc. 140, 1292 (1993)

18. L.C. Nagle, L.D. Burke, J. Solid State Electrochem. 14, 1465 (2010)

19. A. Czerwiński, G. Maruszczak, M. Żelazowska, M. Łańcucka, R. Marassi, S. Zamponi, J. Electroanal. Chem. 386, 207 (1995) 
20. M. Czauderna, G. Maruszczak, A. Czerwiński, J. Radioanal. Nucl. Chem. Lett. 199, 375 (1995)

21. A. Czerwiński, J. Frydrych, I. Kiersztyn, Anal. Lett. 29, 2549 (1996)

22. A. Czerwiński, M. Karwowska, K. Hubkowska, Patent Pending, PL P.414861, 2015 Poland

23. F. Cuevas, M. Hirscher, J. Alloys Compd. 313, 269 (2000)

24. D. Barsellini, A. Visintin, W.E. Triaca, M.P. Soriaga, J. Power Sources 124, 309 (2003)

25. R.C. Ambrosio, E.A. Ticianelli, Surf. Coat. Techn. 197, 215 (2005)
26. A. Visintin, E.B. Castro, S.G. Real, W.E. Triaca, C. Wang, M.P. Soriaga, Electrochim. Acta 51, 3658 (2006)

27. K. Hubkowska, M. Łukaszewski, A. Czerwiński, Electrochim. Acta 56, 2344 (2011)

28. P. Millet, M. Srour, R. Faure, R. Durand, Electrochem. Commun. 3, 478 (2001)

29. M. Łukaszewski, M. Soszko, A. Czerwiński, Int. J. Electrochem. Sci. 11, 4442 (2016)

30. K. Hubkowska, M. Łukaszewski, U. Koss, A. Czerwiński, Electrochim. Acta 132, 214 (2014) 